\title{
Functional Characterization of Piezocrystals Monitored under High Power Driving Conditions
}

\author{
Xiaochun Liao ${ }^{1,2}$, Muhammad Sadiq ${ }^{2}$, Tingyi Jiang ${ }^{1}$, Zhihong Huang ${ }^{1}$, Sandy Cochran ${ }^{3}$, \\ ${ }^{1}$ School of Engineering, Physics and Mathematics, University of Dundee, Dundee, DD1 4HN, UK \\ ${ }^{2}$ Institue for Medical Science and Technology (IMSaT), University of Dundee, Dundee, DD2 1FD, UK \\ ${ }^{3}$ School of Engineering, University of Glasgow, Glasgow, G12 8QQ, UK
}

\begin{abstract}
Relaxor-based ferroelectric single crystals such as PMN-PT are known to exhibit high piezoelectric properties compared with conventional piezoelectric ceramic materials, such as PZT-8. With advances in piezoelectric material development, including compositional engineering, single crystals with higher rhombohedral-to-tetragonal phase transition temperature $\left(T_{R T}\right)$, higher coercive field $\left(E_{C}\right)$ and higher mechanical quality factor $\left(\mathrm{Q}_{M}\right)$ have emerged, the principal example being Mn:PIN-PMN-PT. The improvements have opened up a wider application range, including more demanding high power applications where the performance of conventional materials may deteriorate at elevated temperatures resulting from intrinsic loss mechanisms. Characterization of these piezocrystals under practical and active conditions is therefore important, improving understanding of material behavior and facilitating transducer design in finite element analysis for demanding applications. In this paper, we report an active piezoelectric material characterization system that allows high resolution impedance spectroscopy under conditions similar to those experienced by piezoelectric materials in high power ultrasonic applications. The temperature consequent on the drive voltage is adaptively stabilized using a control algorithm. System function has been verified by testing with a Mn:PIN-PMN-PT thicknessextensional plate and functional characterization has been conducted on Generation I, II and III piezocrystals, with detailed analyses and comparisons of performance stability and material property variation with temperature.
\end{abstract}

Keywords- piezocrystals; piezocrystal characterization; functional characterization; behaviour at high power; thermal response

\section{INTRODUCTION}

Relaxor-based ferroelectric single crystal piezoelectric materials ("piezocrystals") have gained huge interest in both academic research and industrial applications [1]. These materials exhibit extraordinary piezoelectric properties, making them suitable for a range of passive and active applications, including in high bandwidth and high sensitivity piezoelectric transducers [2]. The development of piezocrystals over the last two decades has led to three generations, distinguished by composition: Generation (Gen.) I - binary, e.g. PMN-PT; Gen. II - ternary, e.g. PIN-PMN-PT; and Gen. III - doped ternary, e.g. Mn:PIN-PMN-PT. With recent advances in piezoelectric material development, including compositional engineering, piezocrystals with higher rhombohedral-to-tetragonal phase transition temperature $\left(T_{R T}\right)$, higher coercive field $\left(E_{C}\right)$ and higher mechanical quality factor $\left(\mathrm{Q}_{M}\right)$ have been made possible. These properties are particularly attractive for high power applications, such as therapeutic ultrasound transduction and underwater SONAR projection [3]. In such applications, heat generation results from losses through three mechanisms: dielectric loss, piezoelectric loss and mechanical loss. Affected by both high electric field and high self-heating temperature, the piezocrystal properties vary significantly and functional performance may deteriorate.

As shown in Fig.1, when a $10 \times 10 \times 1 \mathrm{~mm}^{3}$ PMN-PT thickness-extensional (TE) plate is driven at $110 V_{p p}$, the temperature increases to more than $130^{\circ} \mathrm{C}$, well beyond $T_{R T}=96^{\circ} \mathrm{C}$ [1]. Therefore, it is essential to characterize such materials in active configurations, allowing better understanding of property variation and facilitating transducer design in finite element analysis for more demanding high power applications. However, most of the characterization methods reported to date are based on passive approaches in which the piezoelectric material samples are exposed to externally applied environmental temperature and uniaxial pressure. Characterization based on active approaches has also been reported, where a high electric field is applied at ambient temperature [4, 5]. An effective active characterization system was previously reported, integrating the functions of impedance spectroscopy, infrared thermometry, and laser vibrometry; however, only variations in resonant frequency, vibration velocity, and input power were reported, and material property variations were not considered [6].

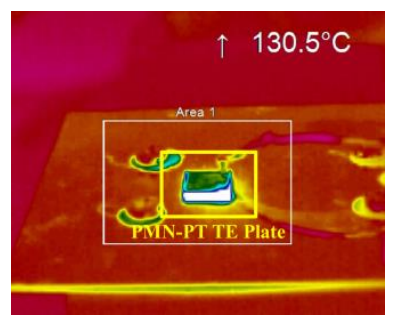

Figure 1. Temperature rise by heat generation in a PMN-PT TE plate with a driving voltage of $110 V_{p P}$

In the study described here, aiming at characterization of variations in the fundamental properties of piezoelectric material in active transduction, we first developed an active piezoelectric characterization system based on high resolution impedance spectroscopy and adaptive temperature stabilization, as explained in Section II. Then, working

Xiaochun Liao holds a SUPA Studentship with funding drawn from the Scottish Funding Council via its SPIRIT initiative. 
conditions were tested in this system to confirm the functions for which it was designed, as presented in Section III. Finally, the system was applied to characterization of three generations of piezocrystals, using thickness-extensional plate samples of PMN-PT, PIN-PMN-PT and Mn:PINPMN-PT. Performance stability and material property variation are compared across the three generations of piezocrystal in Section IV, and conclusions and suggestions for future work are presented in Section V.

\section{ACTIVE PIEZOELECTRIC CHARACTERIZATION SYSTEM}

\section{A. Functional Integration}

The main components in the bespoke active piezoelectric characterization system are an arbitrary waveform generator (33220A, Agilent Technologies, Santa Clara, CA, USA); a radio-frequency $(\mathrm{RF})$ power amplifier (31002A, Electronics \& Innovation, Rochester, NY, USA); a thermal imaging camera (TIM 160, Micro-Epsilon Ltd, Birkenhead, UK); and a PC-based PXIe-1082 system (National Instruments, Newbury, UK), as shown in Fig. 2. Impedance spectroscopy was implemented by recording data measured with a 10:1 passive voltage probe (N2862B, Agilent Technologies, Santa Clara, USA) and a current probe (P6021A, Tektronix, Plano, USA), including necessary fixture compensation, as used in commercial impedance analyzer designs. Material properties and their respective variations were determined from the acquired electric impedance spectroscopy, based on the IEEE standard [7].

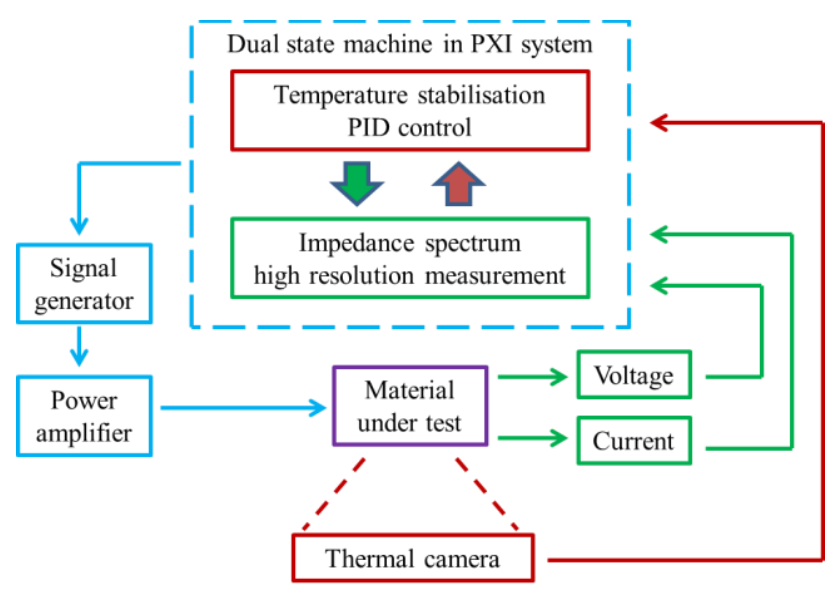

Figure 2. System diagram of active piezoelectric characterisation

\section{B. High resolution impedance spectroscopy}

The objective of active characterization is to measure the impedance spectrum of the piezoelectric sample when it is driven under high electric field at a set temperature, so as to assess the property variation under this active condition. The temperature can vary even when the piezoelectric sample is driven under a constant driving voltage. To maintain the electric field-induced temperature during impedance measurement, the PXI system was programmed with two modules for (1) temperature stabilization (TS) and (2) high resolution impedance spectroscopy (HRIS). The TS module maintains the sample's temperature at a set value while the HRIS module handles measurement of the impedance curve. During temperature stabilization, the whole impedance curve is measured section by section, as shown in Fig. 3. The impedance is calculated from the measured voltage and current, and thus large numbers of measurement points can be achieved for HRIS.

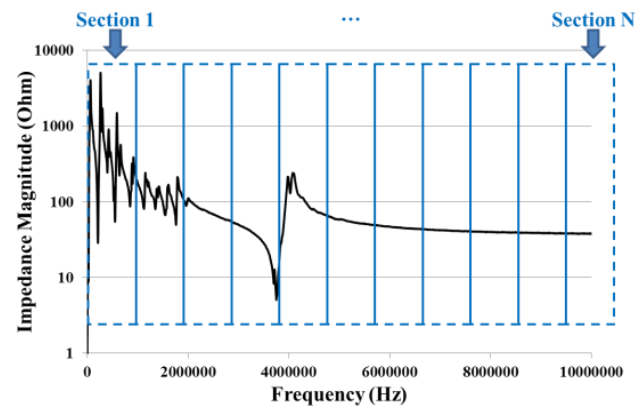

Figure 3. High resolution impedance spectroscopy implemented by making measurements in several sections

\section{Temperature stabilisation with adaptive driving}

The HRIS module is programmed to initiate when the temperature had been maintained at the set value. During impedance measurement, the temperature is monitored in real-time using the thermal imaging camera and the information is fed back to the system. In case of any variation in the temperature data, the program terminates the HRIS module automatically and restarts the TS module. During the whole measurement process, the two states thus switch automatically until the impedance measurement is complete across the full frequency range, as shown in Fig. 4.

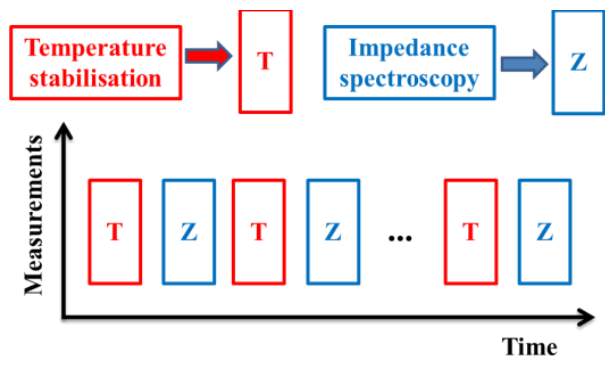

Figure 4. Dual state transition between temperature stabilisation and high resolution impedance spectroscopy shown over time

Temperature stabilization is realized by adaptively adjusting the voltage and the driving frequency to track the piezoelectric sample's resonance, as reported previously [8, 9]. This is achieved with a PID controller programmed in LabVIEW in the PXI system with Eqns 1 and 2:

$$
\begin{aligned}
& f(k)=f(k-1)+\Delta f(k) \\
& \Delta f(k)=k_{p f} \Delta \theta(k)+k_{i f} \Sigma \Delta \theta(j)+k_{d f}[\Delta \theta(k)-\Delta \theta(k-1)] \\
& V(k)=V(k-1)+\Delta V(k) \\
& \Delta V(k)=\left\{k_{p v} \Delta T(k)+k_{i v} \Sigma \Delta T(j)+k_{d v}[\Delta T(k)-\Delta T(k-1)]\right\}|Z|
\end{aligned}
$$

where $f(k)$ is the driving frequency, $\Delta f(k)$ is an incremental adjustment, $\theta(k)$ is the measured impedance phase, $V(k)$ is the driving voltage, $\Delta T(k)$ is the difference between the expected and measured temperatures; $k_{p f}, k_{i f}$, and $k_{d f}$ are proportional, integral and differentiation coefficients for frequency adjustment; and $k_{p v}, k_{i v}$, and $k_{d v}$ are proportional, 
integral and differentiation coefficients for voltage adjustment.

\section{SYSTEM WORKING CONDITIONS}

After implementation of the active piezoelectric characterization system, function verification experiments were conducted with a $5 \times 5 \times 0.5 \mathrm{~mm}^{3} \mathrm{Mn}$ :PIN-PMN-PT TE plate mounted on a spring-loaded fixture as shown in Fig. 5(a). The experimental objective was to measure the TE plate's impedance curve in the frequency range of $10 \mathrm{~Hz}-$ $10 \mathrm{MHz}$ with 800 points at a stabilized temperature of $80^{\circ} \mathrm{C}$. The thermal response was recorded with the thermal imaging camera and used as feedback for the adaptive control of the driving voltage, as shown in Fig. 5 (b).
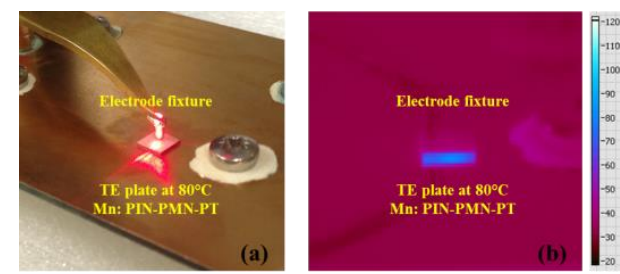

Figure 5. (a) Mn: PIN-PMN-PT TE plate mounted on a spring-loaded fixture and (b) monitored with a thermal imaging camera

The temperature tolerance limit was initially set in the range $75-85^{\circ} \mathrm{C}$. During adaptive driving, the sample temperature was controlled between a lower bound, $76.5-77.8^{\circ} \mathrm{C}$, and an upper bound, $80.0-80.2^{\circ} \mathrm{C}$, for impedance measurement. The driving voltage was adjusted adaptively in the range 5.0 $-17.5 \mathrm{~V}$, during the whole measurement time of $250 \mathrm{sec}$., as shown in Fig. 6.

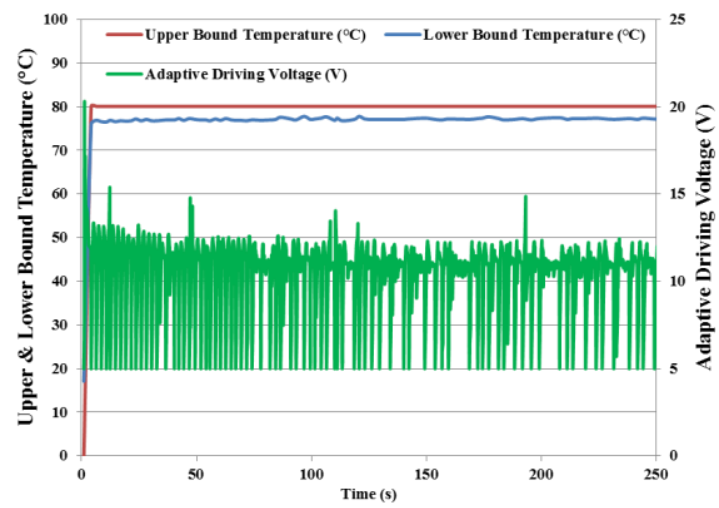

Figure 6. Upper and lower bound temperature during stabilisation with adaptively adjusted driving voltage

The impedance curve was measured with a constant driving voltage of $5 \mathrm{~V}$ from the power amplifier, with frequency swept from $10 \mathrm{~Hz}$ to $10 \mathrm{MHz}$ by the signal generator. Minimum voltage and maximum current peaks were experienced at the sample resonance, and maximum voltage and minimum current peaks at the sample antiresonance, as shown in Fig. 7 (a). Spikes in the voltage and current responses indicated the starting points in each period of HRIS measurement, following previous periods of temperature stabilization. Despite of the transient spikes in voltage and current, the measured impedance was smooth, as shown in Fig. 7 (b) before fixture compensation.
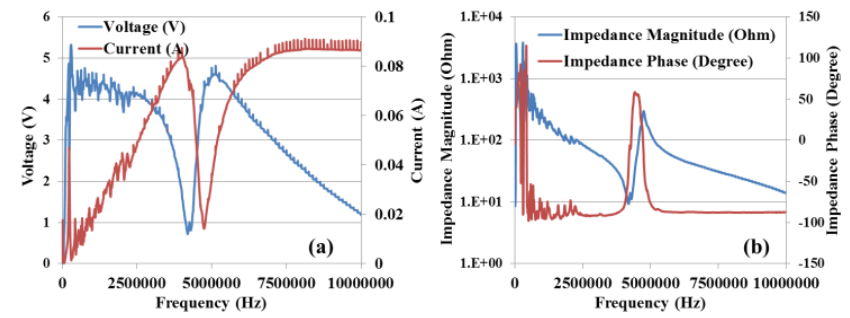

Figure 7. (a) Voltage and current sweep in sections for measurement of (b) impedance magnitude and phase of piezoelectric material

\section{CHARACTERISATION OF PIEZOCRYSTALS}

Utilizing the characterization system, $5 \times 5 \times 0.5 \mathrm{~mm}^{3}$ TE-mode plates of Generations I, II and III piezocrystals were characterized at five stabilized temperatures $T \in\left\{40^{\circ} \mathrm{C}\right.$, $\left.60^{\circ} \mathrm{C}, 80^{\circ} \mathrm{C}, 100^{\circ} \mathrm{C}, 120^{\circ} \mathrm{C}\right\}$. To achieve these temperatures by self-heating, different driving voltages were applied, with $V_{p}$ indicating the maximum voltage during the adaptive driving periods as shown in Table I. In this table, the values highlighted in yellow led to possible piezocrystal phase transitions i.e. when the impedance peaks changed significantly as shown, e.g. at $80^{\circ} \mathrm{C}$ for PMN-PT in Fig. 8, while the values highlighted in green led to depolarization of the piezocrystal samples i.e. when impedance peaks disappeared, e.g. as shown at $100^{\circ} \mathrm{C}$ in Fig. 8. The temperature values recorded at depolarization were $100^{\circ} \mathrm{C}$ and $120^{\circ} \mathrm{C}$ for PMN-PT and PIN-PMNT-PT respectively, which are lower than the depolarization temperature values of $135^{\circ} \mathrm{C}$ and $191^{\circ} \mathrm{C}$ reported in literature [1]. This may be caused by a combination of high temperature and high electric field.

TABLE I. MAXIMUM VOLTAGE $V_{P}$ FOR DIFFERENT OPERATING TEMPERATURES FOR THREE GENERATIONS OF PIEZOCRYSTALS

\begin{tabular}{|c|c|c|c|}
\hline \multirow{2}{*}{$T$} & Mn: PIN-PMN-PT & PIN-PMN-PT & PMN-PT \\
\hline & $V p$ & $V p$ & $V p$ \\
\hline $40{ }^{\circ} \mathrm{C}$ & $5 \mathrm{~V}$ & $11 \mathrm{~V}$ & $10 \mathrm{~V}$ \\
\hline $60{ }^{\circ} \mathrm{C}$ & $9 \mathrm{~V}$ & $16 \mathrm{~V}$ & $13 \mathrm{~V}$ \\
\hline $80{ }^{\circ} \mathrm{C}$ & $12 \mathrm{~V}$ & $20 \mathrm{~V}$ & $20 \mathrm{~V}$ \\
\hline $100{ }^{\circ} \mathrm{C}$ & $13 \mathrm{~V}$ & $14 \mathrm{~V}$ & $30 \mathrm{~V}$ \\
\hline $120^{\circ} \mathrm{C}$ & $13 \mathrm{~V}$ & $42 \mathrm{~V}$ & $32 \mathrm{~V}$ \\
\hline
\end{tabular}

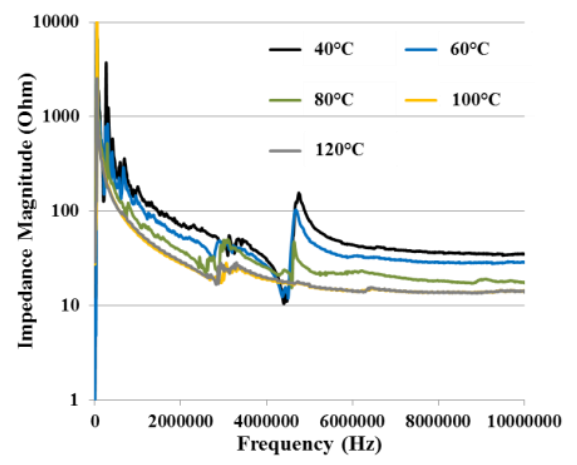

Figure 8. Measured impedance magnitude of a PMN-PT TE plate at different operating temperatures

Four key properties $k_{t}, \varepsilon_{r 33}{ }^{S}, e_{33}$, and $c_{33}{ }^{D}$ were calculated using the IEEE standards [7], based on the impedance 
measurements of the piezocrystal TE plates under different electric field-induced operating temperatures. As shown in Fig. 9, all three generations of piezocrystals followed similar trends. Electromechanical coupling coefficient, $k_{t}$, and piezoelectric constant, $e_{33}$, decreased as the phase transition happened; clamped permittivity, $\varepsilon_{r 33}{ }^{S}$, increased with temperature; and the stiffness constant, $c_{33}{ }^{D}$, decreased with increasing temperature.
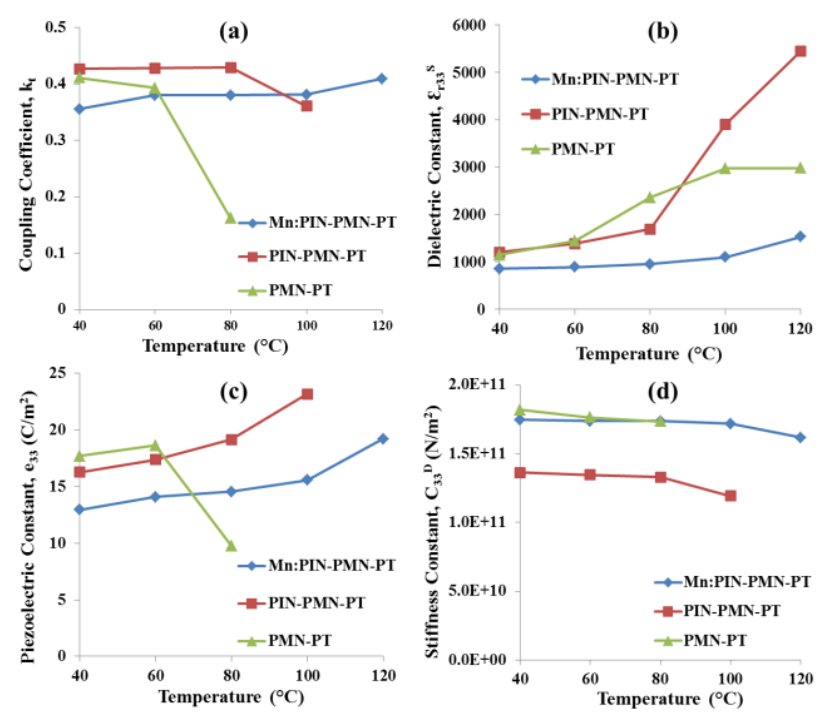

Figure 9. Material property variation in (a) $k_{t}$, (b) $\varepsilon_{r 33}{ }^{S}$, (c) $e_{33}$, and (c) $c_{33}{ }^{D}$ over different operating temperatures for PMN-PT, PIN-PMN-PT and Mn:PIN-PMN-PT

To assess the performance stability at field-induced elevated temperatures, variations in the key properties were compared among the three generations of piezocrystals, as shown in Fig. 10. Generally the third generation Mn:PINPMN-PT exhibited the smallest variations, compared with PIN-PMN-PT and PMN-PT. One issue of note is that $k_{t}$ was slightly lower than expected value of 0.58 [10] for the Mn:PIN-PMN-PT sample, which may have been caused by improper usage or handling of this sample before the test. Despite this defect, stable behavior was still observed at different temperatures for the Mn:PIN-PMN-PT TE sample.

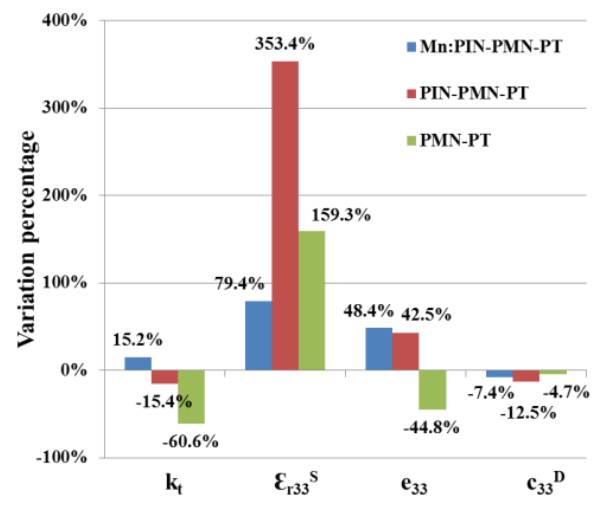

Figure 10. Variation percentage of $k_{t}, \varepsilon_{r 33}{ }^{S}, e_{33}$, and $c_{33}{ }^{D}$ for Mn:PIN-PMNPT, PIN-PMN-PT and PMN-PT

\section{V.CONCLUSIONS AND FUTURE WORK}

This paper has reported the development of an active piezoelectric characterization system with high resolution impedance spectroscopy and adaptive temperature stabilization to characterize piezoelectric materials for high power ultrasound applications. Characterization of three generations of piezocrystals based on performance stability and material property variation confirmed higher stability for Mn:PIN-PMN-PT sample and demonstrated that the characterization system is useful for active functional characterization. Future work will explore integration with laser vibrometry and further use of the system.

\section{ACKNOWLEDGMENTS}

The authors thank the SFC-funded SUPA INSPIRE project for support of studentship. This work has been supported by UK EPSRC project of EP/K020013.

\section{REFERENCES}

[1] S. Zhang, et al., "Advantages and challenges of relaxor-PbTiO3 ferroelectric crystals for electroacoustic transducers - A review," Progress in Materials Science, vol. 68, pp. 1-66, 2015.

[2] S. Zhang and F. Li, "High performance ferroelectric relaxor-PbTiO3 single crystals: Status and perspective," J.Appl.Phys., 111, p. 031301, 2012. [3] E. Sun and W. Cao, "Relaxor-based ferroelectric single crystals: Growth, domain engineering, characterization and applications," Progress in Materials Science, vol. 65, pp. 124-210, 2014.

[4] P. Finkel, et al., "Study of phase transitions in ternary lead indium niobate-lead magnesium niobate-lead titanate relaxor ferroelectric morphotropic single crystals," Appl.Phys.Lett., vol. 97, p. 122903, 2010.

[5] E. A. McLaughlin, et al., "Relaxor ferroelectric PMN-32\%PT crystals under stress, electric field and temperature loading: II-33-mode measurements," Acta Materialia, vol. 53, pp. 4001-4008, 2005.

[6] Z. Yuan, et al., "Loss Factor Characterization Methodology for Piezoelectric Ceramics," IOP Conference Series: Materials Science and Engineering, vol. 18, p. 092027, 2011.

[7] IEEE, "IEEE Standard on Piezoelectricity," ANSI/IEEE Std 176-1987, p. 0_1, 1988 .

[8] L. Xiaochun, et al., "Performance optimization of ultrasonic needle actuating device for insertion operation into tissue mimics," in Ultrasonics Symposium (IUS), 2014 IEEE International, 2014, pp. 823-826.

[9] G. Lin, et al., "Automatic frequency tracking system for needle actuating device," in Ultrasonics Symposium (IUS), 2014 IEEE International, 2014, pp. 815-818.

[10] J. Luo, et al., "A high Qm relaxor ferroelectric single crystal: Growth and characterization," in Ultrasonics Symposium (IUS), 2010 IEEE, 2010, pp. 68-71. 